\title{
CONCEITOS FUNDAMENTAIS DA ANÁLISE DE TERRENOS ANTROPOGÊNICOS: O ESTUDO DA AGÊNCIA GEOLÓGICO-GEOMORFOLÓGICA HUMANA E DE SEUS REGISTROS
}

\author{
Alex Ubiratan Goossens PELOGGIA
}

\begin{abstract}
RESUMO
Apresentam-se neste trabalho as questões conceituais fundamentais referentes ao campo que denominamos Análise de Terrenos Tecnogênicos, que envolve o estudo de processos, depósitos e modelados criados direta ou indiretamente pela agência geológico-geomorfológica humana, caracterizando um enfoque multidisciplinar que, conquanto fundamentado nas Ciências da Terra, dialoga fortemente com as Humanidades. Esta abordagem teórica visa fornecer os subsídios metodológicos para o estudo da época geológica contemporânea, o Antropoceno, bem como dos registros antropogênicos quaternários (holocênicos e, eventualmente, pleistocênicos).
\end{abstract}

Palavras-chave: Terrenos antropogênicos (tecnogênicos ou artificiais); Antropoceno.

\section{ABSTRACT}

\begin{abstract}
FUNDAMENTAL CONCEPTS OF ANALYSIS OF THEANTHROPOGENIC GROUND: THE STUDY OF HUMAN GEOLOGICAL-GEOMORPHOLOGIC AGENCY AND ITS RECORDS. This paper presents the fundamental conceptual issues related to the field called Technogenic Ground Analysis, which involves the study of processes, deposits, and landscapes created directly or indirectly by the human geological-geomorphological agency, adopting a multidisciplinary approach that, although based on Earth Science, is strongly related to the Humanities. This theoretical approach aims to provide the methodological foundation for the study of the current geological epoch, the Anthropocene, as well as of the anthropogenic Quaternary (Holocene and possibly Pleistocene) records.
\end{abstract}

Keywords: Anthropogenic (technogenic or artificial) ground; Anthropocene.

\section{INTRODUÇÃO}

Este ensaio foi produzido como uma contribuição introdutória aos artigos do volume especial sobre o Antropoceno que ora a Revista do Instituto Geológico apresenta. Trata-se de uma revisão teórica acerca dos principais conceitos que, no entendimento deste autor, têm influenciado as pesquisas acerca dos processos e registros geológicos e geomorfológicos da atividade humana, com referência àquelas realizadas no Brasil principalmente, mas também ao redor do mundo. Deve ser ressaltado que as proposições abordadas resultam da iniciativa e da colaboração de diversos cientistas da área das Geociências ao longo de várias décadas, dentre os quais se inclui este pesquisador, e se encontram amplamente referenciadas na literatura.

Dessa forma, ao longo do texto serão citados apenas alguns trabalhos que o autor considera particularmente interessantes como referência na formação do corpo de conhecimento referido, bem como sínteses parciais realizadas anteriormente pelo próprio autor e colaboradores, e que são apresentados como sugestões de leitura, sem nenhuma intenção de uma revisão exaustiva. Em relação a tal incompletude, o autor tem a seu favor apenas a convicção de que as referências citadas suprirão um rol bibliográfico bastante completo e atualizado sobre o tema.

A proposição e disseminação do conceito do Antropoceno (CRUTZEN \& STOERMER 
2000, CRUTZEN 2002), como uma época geológica cuja originalidade consiste na atuação de um novo agente, a humanidade, e que implica em uma nova configuração do Sistema-Terra, tem trazido desafios epistemológicos cuja abrangência engloba as Geociências e as Ciências Ambientais, com reflexos em áreas como a História, a Arqueologia, a Antropologia, a Sociologia e mesmo a Política, a Filosofia e as Artes.

A ideia do Antropoceno, especificamente, surge no contexto das Ciências Ambientais, em essência equivalendo à ideia anteriormente proposta de uma "fase ecológica industrial moderna" (BOYDEN \& HADLEY 1986). O viés estratigráfico (geocronológico) explícito no conceito foi desenvolvido a seguir no âmbito das Geociências, o que implicou em dificuldades significativas, particularmente no que se refere à questão da diacronia do registro antropogênico e da intensidade e abrangência das transformações ambientais associadas. Também é complexo o debate acerca das implicações para a sociedade, e para a relação desta com a natureza, do reconhecimento de se estar vivendo em um tempo geológico gerado pela própria humanidade. Ainda, a discussão tem se aprofundado ao redor do indiscutível caráter necessariamente interdisciplinar desse novo campo, o que se reflete na demanda de colaboração por pesquisadores de diferentes áreas fora das Ciências da Terra.

Todavia, o amplo desenvolvimento teórico dessa discussão, nas décadas de 2000 e 2010, no campo específico das Geociências e em nível internacional (ver para revisão, por exemplo, STEFFEN et al. 2011; RUDDIMAN 2013; WATERS et al. 2014; CEARRETA 2015; HAMILTON \& GRINEVALD 2015; ELLIS 2018; ZALASIEWICS et al. 2018a, 2019a), de certo modo deixou de lado algumas contribuições antecessoras de grande importância, como o conceito de Tecnógeno ou Quinário (TER STEPANIAN 1988), que havia fornecido a base para os estudos da atuação geológica e geomorfológica da humanidade, que no Brasil se desenvolveram a partir da década de 1990, nomeados como "Geologia do Tecnógeno", "Abordagem Geotecnogênica" e "Antropogeomorfologia". Ver, sobre as particularidades de cada abordagem e suas nomenclaturas diferenciadas, por exemplo: OLIVEIRA et al. (2005), OLIVEIRA (2005), PELOGGIA \& OLIVEIRA (2005), RODRIGUES (2005), PELOGGIA et al. (2014a), FRANÇA et al. (2017), PELOGGIA (2017), OLIVEIRA et al. (2018), PELOGGIA (2018a).
Nas Geociências brasileiras se produziu um interessante amálgama entre tais concepções pioneiras e as novas proposições derivadas, internacionalmente, do campo do Antropoceno "propriamente dito", propiciando nos últimos anos o refinamento das concepções originais da década de 1990, a revisão das fontes e o avanço no sentido da compatibilização dos diversos conceitos. Neste trabalho, apresentamos uma síntese do estado da arte desse desenvolvimento, com foco nos principais conceitos disponíveis para o campo de estudos que propomos denominar de Análise de Terrenos Antropogênicos, cujo objeto é a agência geológico-geomorfológica humana e seus registros tecnogênicos. Tal campo se insere nos estudos sobre o Quaternário, de escopo interdisciplinar, e envolve, portanto, os fenômenos e registros de origem humana referentes ao Pleistoceno, ao Holoceno e, desde já considerado, ao Antropoceno como época geológica.

\section{A AGÊNCIA GEOLÓGICA HUMANA E SEUS MÉTODOS DE ESTUDO}

Entendemos que um conceito fundamental que sustenta a particularidade do campo aqui estudado é o de agência (agency, ver ROBB 2005, BARRET 2012, PELOGGIA 2015a, PELOGGIA \& ORTEGA 2016a), proposto em lugar de "ação". A ideia, tomada da Arqueologia Pós-processualista, que por sua vez a incorporou das Ciências Sociais, notadamente de Pierre Bourdieu e Anthony Giddens, é necessária para caracterizar a originalidade da atuação humana e sua diferenciação dos "agentes naturais", cuja ação se dá em função de leis físicas ou de mecanismos puramente biológicos.

Em síntese, a agência (e não simplesmente ação) implica não só relações simples de causa e efeito, processo e resultado, mas um complexo contexto de circunstâncias, determinações, opções e ocorrências imponderáveis, tanto naturais como culturais, que levam indivíduos ou grupos sociais a relacionarem-se de modo particular com os suportes ecológicos e paisagísticos, resultando em processos que os reconfiguram em termos geológicos e geomorfológicos.

A especificidade da agência humana, portanto, envolve a consideração de fatores teleológicos, isto é, referentes à intencionalidade, inserindo assim a dimensão do psíquico, do racional e consciente, mas também do inconsciente, na forma como os seres humanos, genericamente, interfe- 
rem nos processos geológicos e geomorfológicos naturais ou criam novos fenômenos. Esta análise implica, portanto, a consideração do papel da dimensão mental dos seres humanos na reconfiguração do planeta.

É esta peculiaridade que dá ensejo a discussões sobre a própria natureza da "época geológica humana", questionando-se acerca do caráter genérico desta, e se não se trataria de uma situação gerada especificamente em função e no contexto da sociedade capitalista industrial globalizada. Seja como for, mesmo no contexto do mundo capitalista da produção expandida de mercadorias e da exploração exponencial de recursos naturais, seus críticos mais importantes, Marx e Engels, consideravam a relação dialética da humanidade com a natureza, em princípio e como generalidade, como um dado ontológico primário, isto é, referente ao trabalho. É, no entanto, certo que a agência geológica humana concretiza-se em formações sociais específicas, histórica e geograficamente posicionadas e se dá por meio das ferramentas tecnológicas disponíveis (ver PELOGGIA 1995, 1997, 1998, 2004).

A consideração da particularidade da agência geológico-geomorfológica humana, entretanto, tem mostrado ser compatível com o método de estudo do Quaternário proposto por AB'SABER (1969). Esta adaptação envolve três níveis de análise: a) os processos; b) o registro estratigráfico; c) a configuração (modelado) da paisagem. A cada um deles se associam conceitos particulares, que serão analisados neste trabalho. Ao conjunto desses níveis de ação transformadora atribui-se a de- nominação geotecnogênese, que pode ser dividida para finalidade analítica, visto que não ocorrem independentemente, em uma tecnogênese dos processos, uma morfotecnogênese e uma tecnogênese dos depósitos ou estratigráfica (PELOGGIA \& OLIVEIRA 2005).

É de interesse notar que o conhecimento produzido pela análise de terrenos antropogênicos é baseado, antes de tudo, na observação da realidade em campo, como todo procedimento geológico básico. O método fundamental é a análise morfoestratigráfica, que permite a correlação entre as formas de relevo antropogênicas e seus processos de formação com o registro deposicional correlativo. Esta abordagem é complementada por procedimentos geológicos e geomorfológicos usuais, de coleta e análise de material, datação, mapeamento etc., mas também recorre a fontes históricas, iconográficas e literárias, ou mesmo verbais, o que a aproxima dos métodos arqueológicos.

Nesse contexto, temos incluída a possibilidade de uso de obras de arte como referência para a discussão de fenômenos paisagísticos, de natureza geológico-geomorfológica (ver PELOGGIA 2018d para uma discussão aprofundada). Vejamos, por exemplo, uma obra do artista flamengo Hieronymus Bosh, o "Tríptico da Epifania", produzido entre 1485 e 1500, do acervo do Museu do Prado, em Madrid (Figura 1). No quadro se observa, ao fundo, na paisagem a representação de uma cidade que poderíamos chamar de "futurista", em relação aos padrões do século XV. Todavia, o que nos chama a atenção é o plano imediatamente à frente da área urbanizada, que representa uma planície em
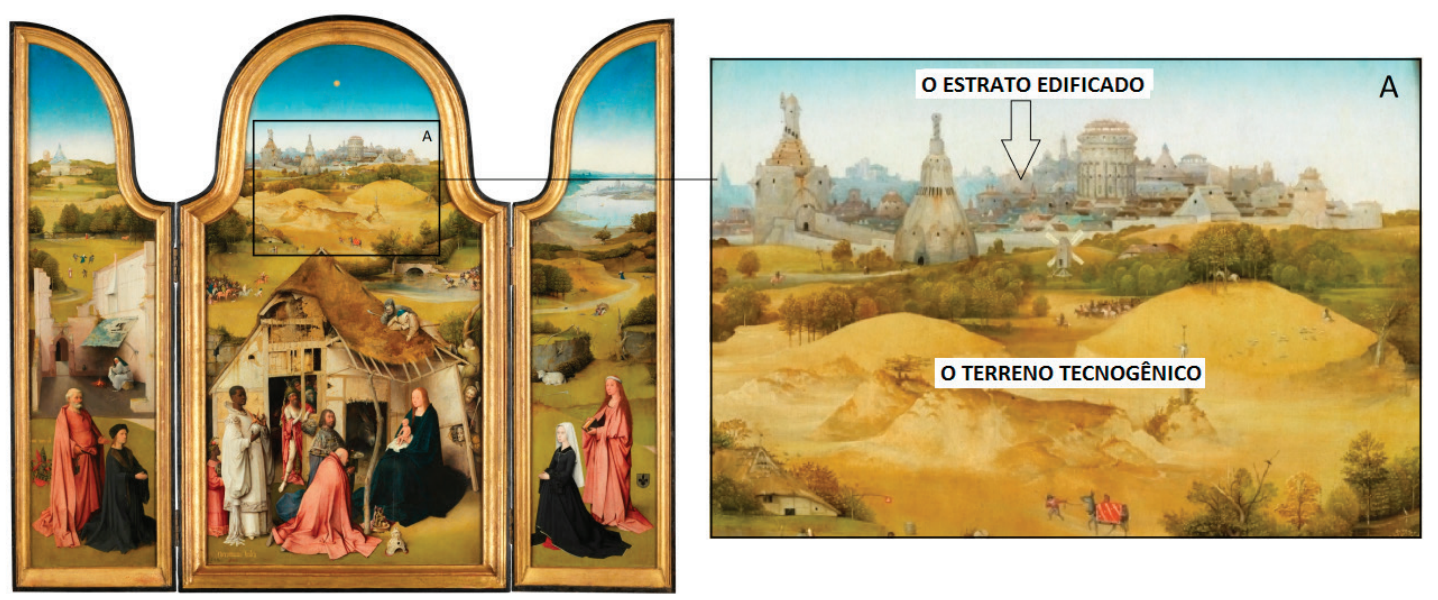

FIGURA 1 - O "Tríptico da Epifania” de Bosh (Século XV), com o detalhe da paisagem ao fundo em que se pode distinguir o estrato urbano edificado e os terrenos tecnogênicos periurbanos. Acervo do Museu do Prado, Madrid. Obra em domínio público. 
que é evidenciada nitidamente a ocorrência de processos antropogênicos de mobilização de material geológico. A interpretação geológica da representação artística nos leva então a associar os dois ambientes: a planície arenosa tecnogênica periurbana, fonte de material geológico para a construção das edificações da cidade, um ambiente geológico em que a extração da matéria prima configurou terrenos artificiais, notadamente do tipo de escavação. É justo imaginar que, sob o estrato construído ao fundo (cujo estatuto estratigráfico ainda está para ser discutido) tenha se formado também um terreno artificial, uma camada de terreno produzido ou construído, como o made ground descrito no início do século XX por Robert Sherlock, para Londres, ou o schuttdecke ("cobertor de detritos"), tal como mapeado por Eduard Suess, ainda no século XIX, em Viena.

Outro exemplo significativo é a tela "Nós Estamos Produzindo um Novo Mundo" (We are Making a New World) (Figura 2), do pintor britânico Paul Nash e integrante do acervo do Imperial War Museum de Londres, produzida em 1918 a partir das experiências do artista na I Guerra Mundial, e cujo sugestivo título, conquanto possivelmente tenha um significado histórico-sociológico, apresenta uma evidente referência paisagística antropogênica. Observa-se no quadro a paisagem produzida como resultado de um aspecto particular da agência humana, as operações de guerra (warfare), especificamente o resultado do impacto dos obuses na "terra de ninguém" (no man's land) entre as linhas de trincheiras. O bombardeamento produziu, então, um terreno tecnogênico caracterizado pela movimentação (revolvimento) da camada superficial de material geológico (disturbed ground), com a formação de um modelado característico em função das crateras decorrentes das explosões (bombturbation).

Como se vê, a fonte artística tem um potencial informativo comparável à literatura, em prosa ou mesmo poesia (ver, sobre esta última abordagem, DONOSO ACEITUNO 2018). Tomemos como exemplo literário em prosa um trecho de Euclides da Cunha, no artigo "As Catas", de 1894, anterior portanto à pioneira referência sobre o Homem como agente geológico feita pelo mesmo autor em "Os Sertões":

\section{“(...) desoladas e ermas, as velhas} Catas mineiras rodeadas pelo silêncio majestoso dos sertões. /.../ Vastas, tintas pelo vermelho duro do Óxido de Ferro que as cobre, envoltas pelos últimos lampejos do poente, com as suas profundas erosões, cortes a prumo, bizarros acidentes

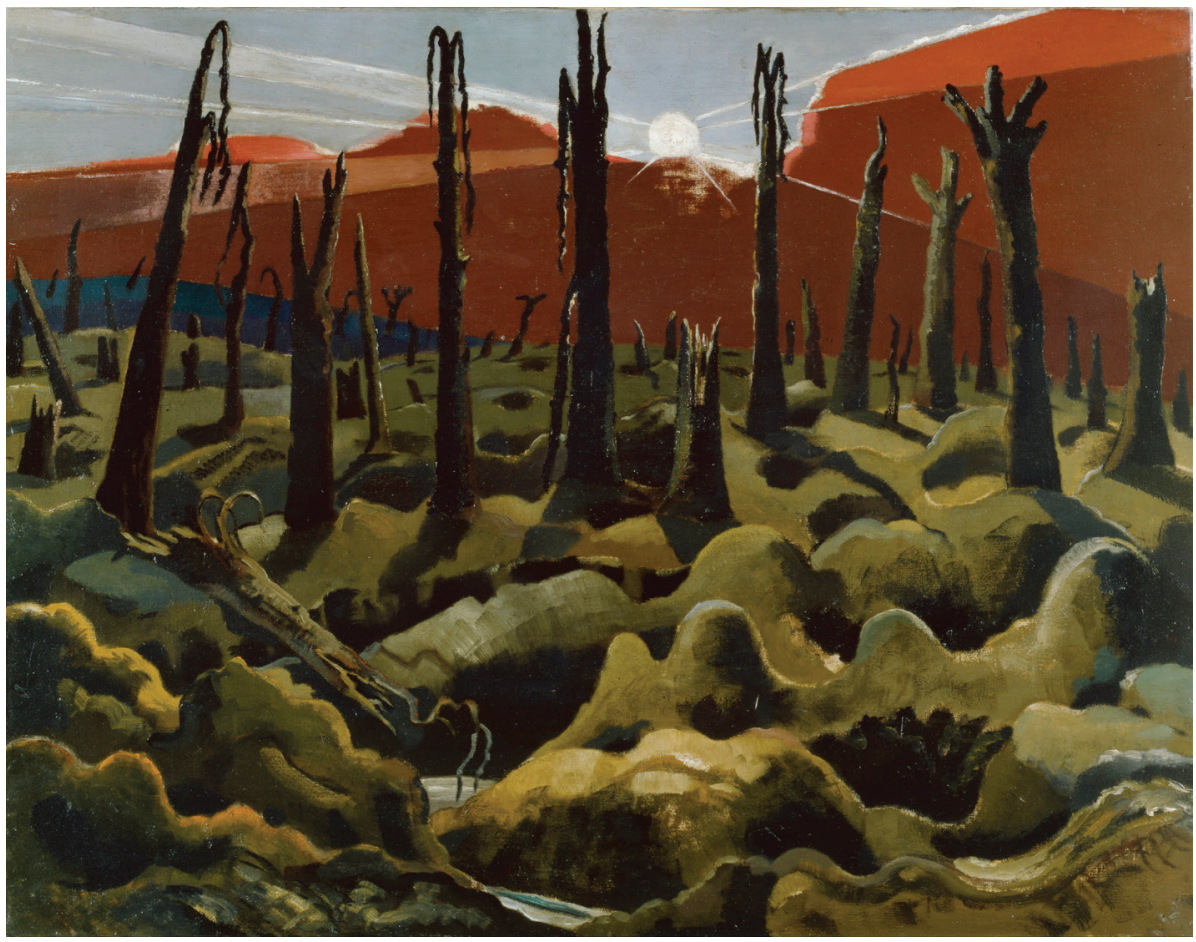

FIGURA 2 - "Estamos Produzindo um Novo Mundo", de Paul Nash (1918). Acervo do Imperial War Museum, Londres. Obra em domínio público. 
derramando-se pelas encostas de colinas afastadas - patenteavam aspecto belíssimo e grandioso." (CUNHA 2018[1894], p.74-75)

Tal descrição de um modelado tecnogênico, acentuada em sua expressividade pelo característico estilo euclidiano (como também fez em relação ao Vale do Paraíba), mostra que, na região considerada (Minas Gerais) a paisagem atual e a estratigrafia devem ser estudadas com a perspectiva de o fator antropogênico ser decisivo em sua formação, mesmo que obliterado pelos processos geomorfológicos superimpostos ao longo dos dois últimos séculos. Por meio de duas vias diferenciadas de expressão, Bosh e Cunha capturam fenômenos comparáveis, relativos ao mesmo objeto: a transformação imposta ao ambiente geológico pela agência humana.

\section{PROCESSOS E REGISTROS GEOLÓGICOS E GEOMORFOLÓGICOS ANTROPOGÊNICOS}

A geotecnogênese é produzida basicamente por três tipos de processos: de agradação (acumulação de material geológico), degradação (retirada ou movimentação de material) e transformação no local (alteração das características dos materiais ou sua movimentação). Dentre os processos de agradação ou degradação, importa distinguir aqueles de caráter induzido daqueles produzidos diretamente. Enquanto o primeiro tipo refere-se a transformações ambientais antropogênicas, ou seja, dos suportes ecológicos, que implicam em alterações de intensidade nos processos de erosão e sedimentação, resultando na formação de registros que, de outra forma, não existiriam, o segundo tipo resulta de ações diretas, artificiais, de mobilização e deposição de materiais. Os processos de transformação in situ, por sua vez, relacionam-se com a incorporação a solos ou depósitos preexistentes de novas substâncias, à modificação de suas características físicas (por compactação, por exemplo) ou revolvimento, fenômenos que podem ocorrer também por ação direta ou indireta, intencional ou não.

\section{Os terrenos tecnogênicos}

O nível de análise essencialmente estratigráfico engloba a questão da descrição, nomenclatura e classificação das camadas e feições geradas pela agência humana, ou seja, os registros tecnogênicos. Aqui temos alguns conceitos centrais: terrenos artificiais (artificial ground) depósitos tecnogênicos (technogenic deposits), geodiversidade tecnogênica (technogenic geodiversity) e estrato geológico humano (human geological stratum) ou arqueoesfera (archaeosphere).

O conceito de terreno artificial, também denominado de tecnogênico (technogenic ground), antropogênico (anthropogenic ground) ou modificado pela humanidade (humanly modified ground), é abrangente e sua classificação relaciona-se aos processos de sua formação: os terrenos de agradação são constituídos por depósitos, de caráter induzido, produzido ou construído ou, ainda, remobilizado. Tais camadas podem ser descritas, geologicamente, em função das características de seu material constituinte e de sua forma de ocorrência, o que constitui a essência do conceito de fácies tecnogênica (technogenic facies). Ressalte-se que as características faciológicas são, frequentemente, decisivas na identificação e classificação dos depósitos, associadamente à morfoestratigrafia.

Assim, o termo depósito tecnogênico (CHEMEKOV 1983) especificamente refere-se a acumulações de material geológico resultantes da atividade humana. O conceito foi desenvolvido inicialmente a partir de duas categorias principais, depósitos construídos (produzidos por ação direta) ou induzidos (formados indiretamente, em decorrência de modificações impressas em processos geológicos) (OLIVEIRA 1990). No segundo grupo englobam-se as camadas descritas como aluviões antropogênicos (anthropogenic alluvium), sedimentos de legado (legacy sediments), aluviões pós-assentamento (postsettlement alluvium) ou depósitos tecnogênicosedimentares (technogenic wash) ou, ainda, aluviação induzida (human-induced alluviation) (ver, por exemplo, JAMES 2013, 2018; BROWN et al. 2013; MACKLIN et al. 2014; PELOGGIA 2015b). Já o termo "solo" tecnogênico, e seus equivalentes tecnossolo, antrossolo, solo antrópico ou antropossolo, embora seja um conceito bastante utilizado, tanto no Brasil como internacionalmente, não nos parece adequado para descrever materiais resultantes de deposição, sendo mais adequadamente restrito a materiais pedogênicos transformados em suas características físicas ou químicas. Nesse sentido, tanto os depósitos quanto os solos formam camadas tecnogênicas (technogenic layers), porém geneticamente diferenciadas.

O conceito de terreno antropogênico é na verdade resultante da conjunção entre a pro- 
posta dos terrenos artificiais (artificial ground, ROSEMBAUM et al. 2003, FORD et al. 2010) do Serviço Geológico Britânico com as ideias desenvolvidas no Brasil a partir da década de 1990 (inicialmente por OLIVEIRA 1990), com base no estudo dos depósitos tecnogênicos, e distingue quatro categorias fundamentais, diferenciadas por critério genético: de agradação (formada por depósitos), de degradação (substratos expostos ou movimentados), modificados (solos tecnogênicos) e complexos (ver PELOGGIA et al. 2014a; PELOGGIA 2017, 2018a; WATERS 2018; OLIVEIRA et al. 2018).

Tais categorias englobam, portanto, tanto o registro sedimentar direta ou indiretamente produzido pela atividade humana, como as formas de relevo resultantes do acúmulo, remoção ou movimentação de material geológico e, ainda, os solos propriamente ditos modificados por ela, sendo o princípio de classificação fundamentado, como vimos, nos processos genéticos que os configuraram. Temos assim sintetizados em um sistema operativo único os três níveis da abordagem metodológica, ou seja, processos, registros correlativos e modelado.

\section{Geodiversidade tecnogênica e patrimônio}

Enfim, a geodiversidade tecnogênica (technogenic geodiversity, PELOGGIA et al. 2014a), isto é, o conjunto dos registros geológicos e geomorfológicos associados às atividades humanas, apresenta algumas características básicas: os depósitos geológicos antropogênicos, são formações superficiais de uma categoria distinta, que podem e têm sido descritas em termos estratigráficos; associam-se em geral a formas de relevo características, o que possibilita seu entendimento por meio de critérios morfoestratigráficos; e seus processos de formação são correlativos à agência humana, ou seja, não só à atuação direta do "agente" humanidade, genericamente colocado, mas também ao modo como esta ação se condiciona e referencia cultural e historicamente, como vimos.

A particularidade da geodiversidade tecnogênica reflete-se na questão de sua proteção e preservação como categoria de patrimônio geológico-geomorfológico, uma vez que se encontra no justo ponto de encontro entre o natural e o cultural, ou melhor, da natureza agenciada pela humanidade, como se refere o Decreto-Lei 25/1937, que instituiu a proteção do patrimônio histórico-cultural no Brasil. A necessidade de sua valorização e proteção, nos mesmos termos da geodiversidade natural, vem sendo colocada por alguns pesquisadores, e se enquadra nos marcos legais já existentes, sem necessidade de diplomas adicionais (ver, para revisão, PELOGGIA 2018c).

O estrato geológico humano ou arqueosfera

Ao longo de milênios, os processos tecnogênicos de agradação têm resultado na formação de uma camada geológica superficial antropogênica que tem se estendido ao longo do planeta, ao menos nos continentes, formando uma camada identificável por correlação geológica: o estrato geológico humano ou arqueosfera, representando o conjunto dos terrenos modificados ou criados pela humanidade, notadamente em processo de desenvolvimento em áreas urbanas (e.g. ZALASIEWICZ 2008, EDGEWORTH et al. 2015, PELOGGIA et al. 2017, ZALASIEWICZ et al. 2017a, EDGEWORTH 2018).

Deve ser enfatizado que se trata aqui de um registro material, frequentemente com uma base bem definida e reconhecível, mas que não se confunde necessariamente com o registro de base sincrônica do Antropoceno "cronogeológico", ao menos se considerarmos as propostas estratigráficas mais recentes, uma vez que se trata de um registro diacrônico. Deve ficar claro que, no atual estágio do desenvolvimento das proposições, as camadas antropogênicas podem ser ou não antropocênicas, e os registros antropocênicos não são necessariamente tecnogênicos.

\section{O PROBLEMA ESTRATIGRÁFICO}

Uma vez que a classificação estratigráfica consiste em uma forma de organização sistemática dos estratos em unidades definidas conforme critérios específicos, e sendo as camadas tecnogênicas materiais geológicos, é justo e adequado que se enquadrem estratigraficamente. Isto, todavia, traz desafios à classificação em virtude da originalidade destes registros (ver revisões em PELOGGIA 2003, WATERS et al. 2014, FORD et al. 2014). Basicamente, a discussão se referencia a dois grandes temas: o temporal e o material.

\section{Cronoestratigrafia/geocronologia}

A questão relativa ao registro temporal tem gerado polêmicas no meio internacional. Conquanto a proposição de uma unidade cronoestratigráfica com o estatuto de sistema (cujo equivalente geocronológico é a época), com uma base sincrônica, traga problemas importantes no estudo 
interdisciplinar da agência geológica humana (uma vez que o registro material antropogênico tem base diacrônica), trata-se de uma questão de difícil solução. Um ponto central do problema decorreu da adoção "informal e generalizada" do termo Antropoceno nas últimas duas décadas, não só nas Geociências, mas na ciência em geral e na cultura, em detrimento de propostas anteriores, como a do Tecnógeno de TER STEPANIAN (1988). Desde então, o termo Antropoceno tem sido usado em mais de um sentido, sendo tanto entendido como uma fase da história cultural-ecológica humana como uma unidade do tempo geológico. A evolução da discussão estratigráfica pode ser verificada, por exemplo, em PELOGGIA (2003), ZALASIEWICS et al. (2011), WATERS et al. (2014), OLIVEIRA \& PELOGGIA (2014), GIBBARD \& WALKER (2014), LEWIS \& MASLIN (2015), STEFFEN et al. (2016), ZALASIEWICS et al. (2017b, c, d), WATERS et al. (2018), ZALASIEWICZ et al. (2018a,b), ZALASIEWICZ et al. (2019a, b), EDGEWORTH et al. (2019).

Seja como for, a rápida consolidação do termo que, portanto, dificilmente será trocado, legou aos estratígrafos o sufixo "ceno", referente a épocas ou séries geológicas, o que induz à ideia de uma época do período Quaternário posterior ao Holoceno. Essa alternativa, que parece ser a mais provável de formalização atualmente, por um lado tem o mérito de marcar os tempos atuais como o ápice de um processo de transformação da natu- reza pela agência humana que se desenvolveu ao longo do Quaternário, intensificado no Holoceno. Mas, por outro lado, como citado, tem o inconveniente de classificar como pertencentes a épocas diferentes sequências de camadas antropogênicas eventualmente produzidas nos mesmos episódios deposicionais.

Enquadra-se neste caso parte significativa do registro antropogênico associado às cidades, ou seja, os estratos geológicos urbanos, cujos processos de formação e expansão apresentam, assim, condicionantes referentes à configuração dos sítios urbanos originais e da região (relevo, hidrografia, tipo de substrato, clima, cobertura vegetal), mas também decorrem da forma como os povoadores, dotados de diferentes culturas e em momentos históricos diferenciados, se apropriaram da região. $\mathrm{Ou}$ seja, tratam-se de questões de natureza geohistórica, cujos registros estratigráficos locais ou regionais pouco têm a ganhar em seu entendimento com a noção de um Antropoceno global e muito recente.

A classificação estratigráfica do registro material antropogênico

O problema do registro material é de solução mais simples. Levando-se em conta que a classificação estratigráfica deve se adaptar à realidade geológica observada e às necessidades da pesquisa, o problema estratigráfico das camadas antropogênicas centra-se então, em um primeiro momento, na adequação dos conceitos geológicos preexistentes

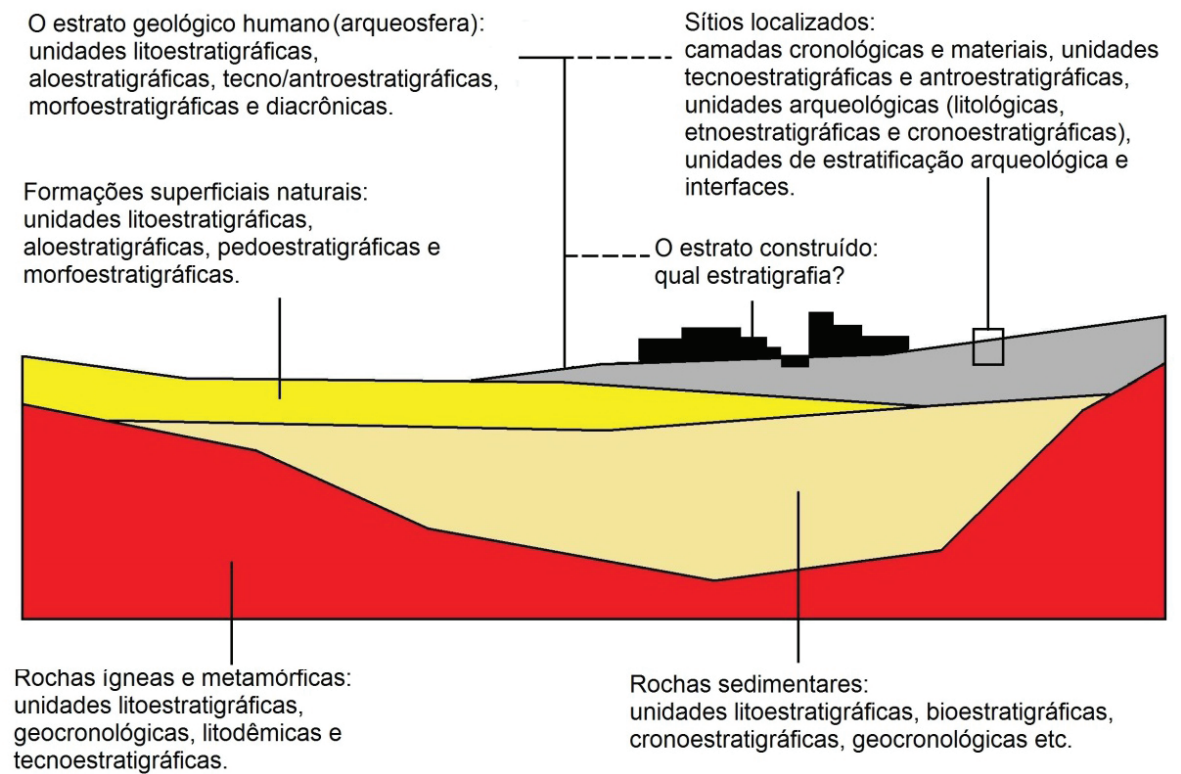

FIGURA 3 - Quadro comparativo da utilização de diferentes abordagens estratigráficas em função do tipo de terreno. 
para sua caracterização. A figura 3 apresenta um esboço comparativo entre os critérios estratigráficos frequentemente utilizados para os diferentes tipos de configurações geológicas naturais, acrescentando-se as possibilidades de abordagem dos terrenos tecnogênicos.

Esta questão está sendo atualmente discutida nos níveis internacional e local, inclusive no âmbito da Comissão Brasileira de Estratigrafia, da Sociedade Brasileira de Geologia, da qual o autor é membro. O problema central consiste essencialmente em duas possibilidades: a utilização, eventualmente adaptada, do quadro de unidades estratigráficas existentes para a caracterização das camadas antropogênicas, ou a eventual criação de unidades diferenciadas para tal aplicação. $\mathrm{Na}$ opinião deste autor, a primeira possibilidade é a mais adequada, sendo possível adaptar o uso de unidades litoestratigráficas (como tem feito o Serviço Geológico Britânico para terrenos constituídos por depósitos construídos, ver FORD et al. 2014) ou aloestratigráficas (como já tem sido usado para camadas tecnogênico-sedimentares induzidas, ver por exemplo PELOGGIA 2015b). Uma dificuldade particular consiste na abordagem geológica do estrato urbano edificado, isto é, não o substrato urbano tecnogênico, mas o conjunto das edificações em si, uma vez que este pode ser e de fato já tem sido comparado a construções biogênicas, como os recifes de coral.

Além disso, parece ter grande potencial o uso de uma abordagem de estratigrafia de eventos, a partir dos moldes propostos pelo Guia Estratigráfico da Subcomissão de Estratigrafia do Quaternário, da Comissão Internacional de Estratigrafia da União Internacional de Ciências Geológicas, e com o uso de unidades estratigráficas diacrônicas, como as previstas no Código Estratigráfico Norte-Americano (NACSN 1983), em termos de uma "estratigrafia de eventos tecnogênicos ou antropogênicos", uma vez que estes teriam a potencialidade de refletir detalhadamente as particularidades locais e regionais e, por correlação geológica, até as globais, da gênese dos registros, abrindo amplas possibilidades de aportes interdisciplinares na abordagem (STINCHCOMB et al. 2013, OLIVEIRA \& PELOGGIA 2014).

Por outro lado, a particularidade da ocorrência de artefatos, isto é, objetos artificialmente produzidos, que têm sido referidos como tecnofósseis (ZALASIEWICZ et al. 2014a), nas camadas quaternárias (antropogênicas ou não), também justifica a consideração de propostas como a tecnoestra- tigrafia, a antroestratigrafia ou a etnoestratigrafia (GASHE \& TUNCA 1983, HOWARD 2014), além de implicar em considerações bioestratigráficas importantes, visto que os artefatos humanos (tecnofósseis) podem, em princípio, ser enquadrados sem dificuldades na categoria mais geral de icnofósseis, isto é, vestígios da atividade de um organismo (no caso, a atividade produtiva do ser humano).

Por fim, mas não menos importante, resta lembrar que o registro paleontológico em si também sofre, no Quaternário, influência significativa da agência humana, em função da introdução de espécies provenientes de seleção artificial e da modificação dos padrões de distribuição biogeográfica (espécies introduzidas ou invasoras), bem como de novos processos tafonômicos (ver, por exemplo, WILLIAMS et al. 2018)

\section{A PAISAGEM ANTROPOGÊNICA}

\section{Formas de relevo tecnogênicas}

A modificação do relevo, com a criação de modelados originais (relevo antropogênico), é o aspecto mais evidente da geotecnogênese, e tem sido indicada há tempos (ver, por exemplo, GOUDIE 1993, 2018; BROWN et al. 2013, 2017; PELOGGIA et al. 2014b; TAROLLI \& SOFIA 2016; LI et al. 2017 e COOPER et al. 2018, para revisões). É interessante notar também que as feições de relevo tecnogênicas (technogenic landforms) frequentemente constituem anomalias na configuração do modelado natural local ou regional, o que facilita a aplicação da análise morfoestratigráfica, como já citado.

A conjunção entre ações diretas ou indiretas envolvendo processos de degradação (erosão ou movimentação) e agradação (deposicão) implica na formação de quatro tipos básicos de superfícies tecnogênicas: escavadas, de acumulação ou preenchimento, erodidas ou movimentadas ou de subsidência e, ainda, de sedimentação induzida. A complexidade da ocorrência dos processos em contextos reais resulta, todavia, na ocorrência de superfícies mistas envolvendo dois ou mais dos tipos básicos (PELOGGIA et al. 2014b).

No entanto, um aspecto fundamental na análise do relevo tecnogênico diz respeito à aplicação do conceito de taxonomia, fundamentado na proposta por ROSS (1992). A associação das superfícies geomórficas tecnogênicas em diferentes escalas implica, então, na definição de unidades morfotecnogênicas representados por tipos de formas 
de relevo tecnogênicas, compartimentos de modelado tecnogênico e paisagens tecnogênicas (ver PELOGGIA et al. 2014b).

\section{Sistemas Tecnogênicos e Tecnosfera}

O conceito de sistema tecnogênico (Technogenic Earth System, FIGUEIRA 2007) foi proposto, essencialmente, para caracterizar conjuntos de processos interligados, de origem estritamente antropogênica ou que ocorrem associadamente a processos naturais, que reconfiguram de maneira particular a dinâmica geológico-geomorfológica em certo meio definido, por um lado, pelas características originais do sítio (ou seja, dos compartimentos geomorfológicos) e, por outro, pelo tipo de intervenção humana predominante (resultante do modelo de apropriação do território). Resulta disso que cada sistema tecnogênico vai apresentar bases geológicas (incluindo-se os terrenos tecnogênicos) e configurações paisagísticas particularizadas. Enfim, o conjunto global dos sistemas tecnogênicos configura o que tem sido denominado de Sistema Terra Humanizado (Humanized Earth System, HAFF 2014) ou Tecnosfera (Technosphere, RULL 2016).

Neste contexto insere-se o conceito de Tecnosfera Física (Physical Technosphere,
ZALASIEWICZ et al. 2017d), englobando os materiais tecnológicos em que componentes humanos podem ser distinguidos, parte dos quais se encontra em uso ativo (o que corresponde ao conceito de contexto sistêmico da Arqueologia, SCHIFFER 1996) e parte constitui resíduos (o contexto arqueológico propriamente dito).

\section{TÓPICOS AVANÇADOS}

Recentemente, o estudo da agência humana sobre o ambiente geológico-geomorfológico tem avançado para a consideração de aspectos que vão além das questões básicas de conceituação e classificação, inserindo-se nos campos do ensino, da geoconservação, dos estudos ambientais, da Arqueologia e da Geologia Aplicada. Alguns exemplos são discutidos a seguir.

O novo ciclo geológico antropogênico

A ocorrência de novos processos geodinâmicos, a produção de novos materiais sintéticos equivalentes a minerais e rochas e a formação de camadas tecnogênicas permite a reconfiguração do conhecido modelo conceitual do ciclo das rochas, ou ciclo geológico, com a inserção desses elementos (Figura 4). Tal ciclo, em que a importância estratigráfica de novos materiais como concreto e plástico

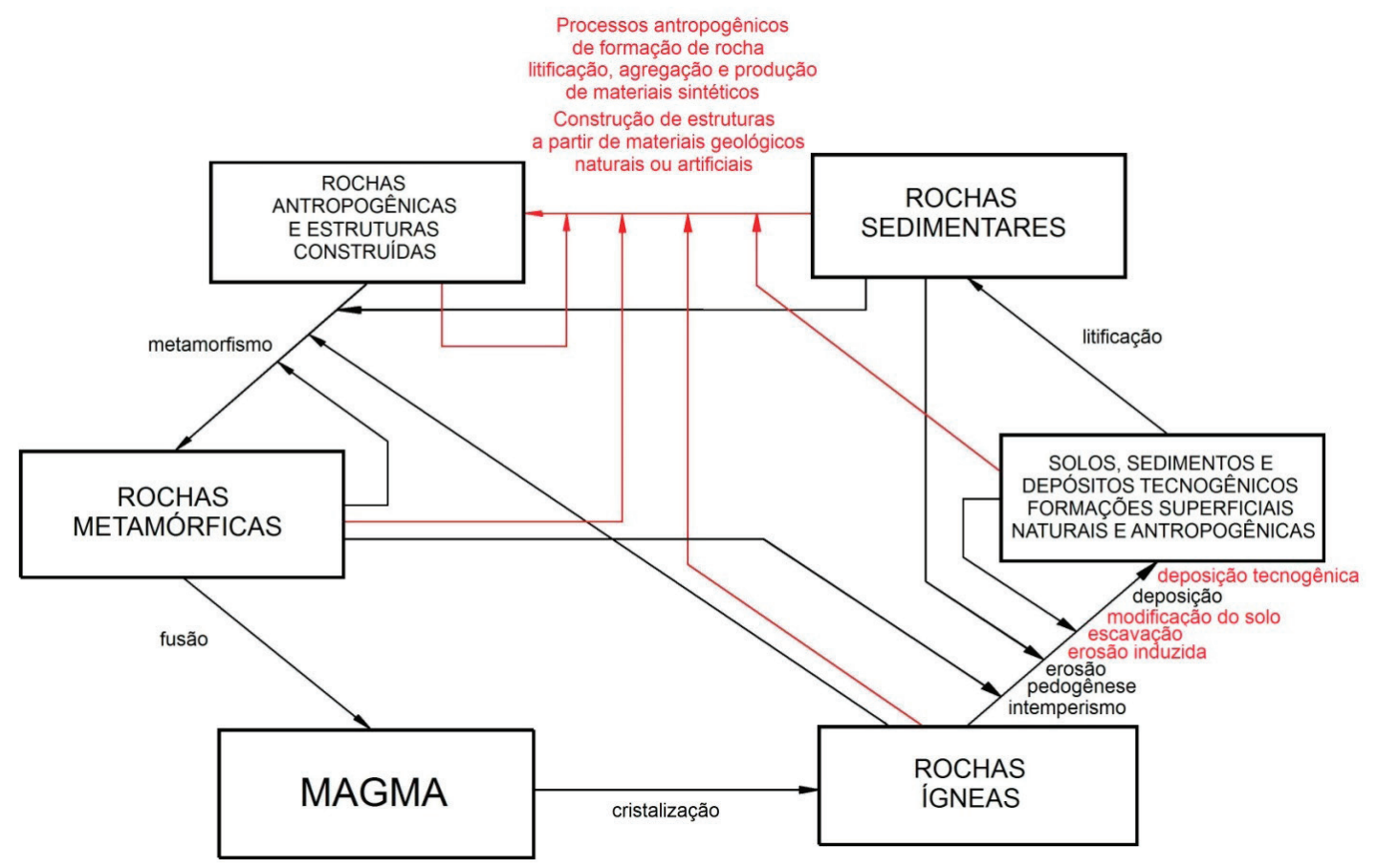

FIGURA 4 - O ciclo geológico antropogênico (adaptado de PELOGGIA 2018b). 
tem sido apontada, vem se configurando progressivamente em função da intensificação da agência geológica humana ao longo do Quaternário, e deve se consolidar como o ciclo típico do Antropoceno. (e.g. UNDERWOOD 2001a, b; CATHCART 2011; ZALASIEWICZ et. al. 2014b, 2016; HAZEN et al. 2017; WATERS \& ZALASIEWICS 2018; PELOGGIA 2018b).

Geoecologia de terrenos tecnogênicos e estudos de impacto ambiental

Os processos tecnogênicos de apropriação do relevo apresentam dois aspectos fundamentais em termos geoecológicos. O primeiro, mais conhecido, é associado a episódios agudos de desequilíbrio ambiental (resistasia antropogênica) ou mesmo, de crise ambiental persistente por períodos históricos de longa duração, como pode ser referido, por exemplo, aos efeitos do Ciclo do Ouro, do Café e da Urbanização no Sudeste do Brasil, entre o século XVIII e a atualidade (e.g. PELOGGIA \& ORTEGA 2016b). Tais fenômenos são responsáveis por impactos paisagísticos intensos e persistentes, que implicam em ampla reconfiguração de sistemas ecológicos. Por outro lado, uma derivação relativamente pouco estudada ainda diz respeito aos processos de "reconstituição" geoecológica e formação de novos ecossistemas, referidos como "antrobiomas" ou "tecnobiomas", bem como de novos solos pedogênicos, em diferentes escalas, desde a global até a local.

Seja como for, uma implicação fundamental derivada da análise de terrenos tecnogênicos é que os estudos de impacto ambiental muito frequentemente trabalham com contextos já muito transformados, por vezes há séculos, pela agência humana, ficando claro que a aplicação de padrões de avaliação vinculados a modelos teóricos estritamente "naturais" (de uma "natureza intocada") e na consideração de "serviços ecossistêmicos" a eles vinculados, muitas vezes não existentes na realidade, terá pouca efetividade, bem como será inadequada, nesses casos, a proposição de ações de recuperação de áreas pautadas em tais modelos.

\section{Geoarqueologia e Arqueologia da Paisagem}

Em princípio, todas as camadas antropogênicas têm significado arqueológico, uma vez que são correlativas à agência humana, mesmo que não sejam associadas a sítios arqueológicos em sentido estrito, ou seja, caracterizados por ocorrências significativas de restos humanos ou vestígios de atividade humana, aos quais têm se associado os termos "camadas culturais" ou "sedimentos arqueológicos".

Uma questão que merece discussão é a adequação entre os métodos estratigráficos geológicos e aqueles utilizados pela Arqueologia para a descrição de sítios específicos, em escalas de superdetalhe, uma vez que estes, na caracterização da sobreposição das "camadas culturais", levam em consideração elementos específicos como estruturas e interfaces.

Ainda neste campo, a análise de terrenos tecnogênicos permite a inserção de uma visão expandida da paisagem e de suas transformações em escalas de trabalho não usuais nas investigações arqueológicas, abordagem que pode contribuir decisivamente na prospecção, além de reforçar o entendimento das camadas tecnogênicas como objetos de interesse em si, independentemente da ocorrência de artefatos ou restos humanos. Seja como for, se faz necessário um trabalho de compatibilização, certamente viável, entre os conceitos geológicos emergentes relativos aos registros antropogênicos e as propostas arqueológicas sobre o mesmo tema (e.g. BORJA BARRERA 1993; ERLANDSON \& BRAJE 2013; HARRIS 2014; EDGEWORTH 2014a, b; PELOGGIA 2015a, b).

\section{Riscos geológicos e gestão de desastres}

Tem sido amplamente mostrado, desde há décadas, que a ocorrência de situações de riscos e desastres geológicos associa-se significativamente à presença de terrenos tecnogênicos, notadamente em áreas urbanas, mas não só. Assim, nas análises de riscos, inclusive naquelas de caráter previsional (que muitas vezes ainda são feitas levando em conta somente indicadores naturais de configuração de relevo e constituição geológica), as configurações tecnogênicas devem consideradas como elementos prioritários, sob a pena de se produzirem estudos de pouca aplicabilidade em função dessa desconsideração metodológica. Esta proposição se insere no conceito de uma análise integrada e multidimensional de riscos.

\section{CONSIDERAÇÕES FINAIS}

O estudo da agência geológico-geomorfológica humana e de seus registros, na forma de camadas geológicas ou formas de relevo e paisagens, e de seus processos de formação, como vimos não se confunde com o estudo do Antropoceno como 
época geológica, embora seja um elemento fundamental deste.

Ressaltamos que a revisão efetuada neste trabalho não é exaustiva, baseando-se em proposições realizadas por diversos pesquisadores, e que representa uma visão de síntese que, embora propiciada por quase três décadas de dedicação do autor ao tema, reflete certamente um ponto de vista pessoal. Esta reflexão não substitui o acesso às contribuições originais disponíveis na literatura, das quais somente algumas foram referenciadas diretamente, como observamos de início, em função do estilo e da natureza didática do texto.

\section{AGRADECIMENTOS}

O autor agradece ao Museu do Prado (Madrid) pela cessão da imagem do Tríptico da Epifania, de Bosh, e ao Imperial War Museum (Londres) pela disponibilização da imagem de We Are Making a New World, de Nash. Agradece também aos editores da Revista do Instituto Geológico pela oportunidade de publicar este ensaio, bem como aos relatores pelas oportunas sugestões.

\section{REFERÊNCIAS}

AB'SABER, A.N. 1969. Um conceito de Geomorfologia a serviço das pesquisas sobre o Quaternário. Geomorfologia (Instituto de Geografia/USP), 18: 1-23.

BARRETT, J.C. 2012. Agency: a revisionist account. In: I. Hodder (ed.) Archaeological theory today. Oxford, Polity Press, $2^{\text {nd }}$ ed., p. 146-166.

BORJA BARRERA, F. 1993. Formaciones superficiales antrópicas: aportación al studio del proceso holoceno de antropización de los sistemas naturales. Arqueologia Espacial, 16-17: 29-39.

BOYDEN, S; HADLEY, M. 1986 The hand of Man. The Unesco Courrier, 7: 35-37.

BROWN, A.; TOMS, P.; CAREY, C.; RHODES, E. 2013. Geomorphology of the Anthropocene: time-transgressive discontinuities of human induced alluviation. Anthropocene, 1: 3-13.

BROWN, A.C.; TOOTH, S.; BULLARD, J.E.; THOMAS, D.S.G.; CHIVERRELL, R.C.; PLATER, A.J.; MURTON, J.;
THORNDYCRAFT, V.R.; TAROLLI, P.; ROSE, J.; WAINWRIGHT, J.; DOWNS, P.; AALTO, R. 2017. The geomorphology of the Anthropocene: emeegence, status and implications. Earth Surface Processes and Landforms, 42(1): 71-90. https://doi. org/10.1002/esp.3943

CATHCART, R.B. 2011. Anthropic rock: a brief history. History of Geo- and Space Sciences, 2: 57-74. http://dx.doi.org/10.5194/hgss-2$57-2011$

CEARRETA, A. 2015. La definición geológica del Antropoceno según el Anthropocene Working Group (AWG). Enseñanza de Las Ciencias de la Tierra, 23(3): 263-271.

CHEMEKOV, Y.F. 1983. Technogenic deposits. In: INQUA, INTERNATIONAL ASSOCIATION FOR QUATERNARY STUDIES, 11, Moscow, Abstracts, v.3, p. 62.

COOPER,A.H.;BROWN, T.J.;PRICE, S.J.;FORD, J.R.; WATERS, C.N. 2018. Humans are the most significant global geomorphological driving force of the $21^{\text {st }}$ century. The Anthropocene Review, 5(3): 222-229. https:// doi.org/10.1177\%2F2053019618800234

CRUTZEN, P. 2002. Geology of Mankind. Nature, 415: 23. https://doi.org/10.1038/415023a

CRUTZEN, P.; STOERMER, E.F. 2000. The "Anthropocene". Global Change Newsletter, 41: 17-18.

CUNHA, E. 2018 [1894]. As catas. In: L.M. Bernucci \& F.P. Rissato (org.) Ensaios $e$ Inéditos. São Paulo, Editora UNESP, p. 7377.

DONOSO ACEITUNO, A. 2018. Imágenes del Antropoceno em la poesía chilena. Anales de Literatura Chilena, 19(30): 205-216.

EDGEWORTH, M. 2014a. Archaeology of the Anthropocene. Journal of Contemporary Archaeology, 1(1): 73-77. http://dx.doi. org/10.1558/jca.v1.i1.73

EDGEWORTH, M. 2014b. The relationship between archaeological stratigraphy and artificial ground and its significance in the Anthropocene. In: C.N. Waters; J.A. Zalasiewicz; M. Willliams; M.A. Elllis; A.M. 
Snelling (eds.) A stratigraphical basis for the Anthropocene. London, Geological Society (Special Publication 395), p. 91-108. https:// doi.org/10.1144/SP395.3

EDGEWORTH, M. 2018. Humanly modified ground. In: D.A. Della Sala \& M.I. Goldstein(eds) The Encyclopaedia of the Anthropocene, Oxford, Elsevier, v.1, p. 157161. http://dx.doi.org/10.1016/B978-0-12409548-9.09999-1

EDGEWORTH, M.; RICHTER, D.B.; WATERS, C.; HAFF, P.; NEAL, C.; PRICE, S.J. 2015. Diachronous beginnings of the Anthropocene: the lower bounding surface of anthropogenic deposits. The Anthropocene Review, 2(1): 33-58. https:// doi.org/10.1177\%2F2053019614565394

EDGEWORTH, M.; ELLIS, E.C.; GIBBARD, P.; NEAL, C.; ELLIS, M. 2019. The chronostratigraphic method is insuaitable for determining the start of the Anthropocene. Progress in Physical Geography (no prelo). http://dx.doi. org/10.1177/0309133319831673

ELLIS, E.C. 2018. Anthropocene: a very short introduction. Oxford University Press, Oxford, $185 \mathrm{p}$.

ERLANDSON, J.M.; BRAJE, T.J. 2013. Archaeology and the Anthropocene. Anthropocene, 4: 1-7. http://dx.doi. org/10.1016/j.ancene.2014.05.003_

FIGUEIRA, R.M. 2007. Evolução dos sistemas tecnogênicos no Município de São Paulo. Instituto de Geociências, Universidade de São Paulo, São Paulo, Dissertação de Mestrado, 126 p. http://dx.doi.org/10.11606/D.44.2007. tde-31072007-113831

FORD, J..; KESSLER, H.; PRICE, S.J.; HUMPAGE, A.J. 2010. An enhanced classification for artificial ground. Keyworth: British Geological Survey, Keyworth, 32 p. (British Geological Survey Open Report OR/10/036).

FORD, J.R.; PRICE, S.J.; COOPER, A.H.; WATERS, C.N. 2014. An assessment of lithostratigraphy for anthropogenic deposits. In: C.N. Waters, J.A. ZALASIEWICZ,. M.
Williams, M.A. Ellis, A.M. SNELLING (eds.) A stratigraphical basis for the Anthropocene. London, Geological Society (Special Publication 395), p. 55-89. https:// doi.org/10.1144/SP395.12

FRANÇA JR., P.; KORB, C.C.; BRANNSTROM, C. 2017. Research on Technogene/ Anthropocene in Brazil. Quaternary and Environmental. Geosciences, 9(1): 1-10. http://dx.doi.org/10.5380/abequa.v9i1.52453

GASCHE H., TUNCA, O. 1983. Guide to archaeostratigraphic classification and terminology; definitions and principles. Journal of Field Archaeology, 10(3): 325-335. https://doi.org/10.1179/009346983791504318

GIBBARD, P.L.; WALKER, M.J.C. 2014. The term 'Anthropocene' in the context of formal geological classification. In: C.N. Waters, J.A. Zalasiewicz, M. Williams, M.A. Ellis, A.M. Snelling (eds.) A stratigraphical basis for the Anthropocene. London, Geological Society (Special Publication 395), p. 29-37. https://doi.org/10.1144/SP395.1

GOUDIE, A. 1993. Human influence in Geomorphology. Geomorphology, 7: 37-59. https://doi.org/10.1016/0169-555X(93)90011-P

GOUDIE, A. 2018. The human impact in geomorphology - 50 years of change. Geomorphology (no prelo). https://doi. org/10.1016/j.geomorph2018.12.002

HAFF, P.K. 2014. Technology as a geological phenomenon: implications for human wellbeing. In: C.N. Waters; J.A. Zalasiewicz; M. Williams; M.A. Ellis; A.M. Snelling (eds.) $A$ stratigraphical basis for the Anthropocene. London, Geological Society (Special Publication 395), p. 301-330. https://doi. org/10.1144/SP395.4

HAMILTON， C.; GRINEVALD， J. 2015. Was the Anthropocene anticipated? The Anthropocene Review, 2(1): 59-72. https:// doi.org/10.1177\%2F2053019614567155

HARRIS, E.C. 2014 Archaeological stratigraphy: a paradigm for the Anthropocene. Journal of Contemporary Archaeology, 1(1): 105-109. http://dx.doi.org/10.1558/jca.v1i1.105 
HAZEN, R.M.; GREW, E.S.; OROGLIERI, M.J.; DOWNS, R.T. 2017. On the mineralogy of the "Anthropocene Epoch". American Mineralogist, 102: 595-611. https://doi. org/10.2138/am-2017-5875

HOWARD, J.L. 2014. Proposal to add anthrostratigraphic and technostratigraphic units to the stratigraphic code for classification of anthropogenic Holocene deposits. The Holocene, 24(12): 1856-1861. https://doi. org/10.1177\%2F0959683614551231.

JAMES, L.A. 2013. Legacy sediment: definitions and processes of episodically produced anthropogenic sediment. Anthropocene, 2: 1626. https://doi.org/10.1016/j.ancene.2013.04.001

JAMES, L.A. 2018. Ten conceptual models of large-scale legacy sedimentation: a review. Geomorphology, 317: 199-217. https://doi. org/10.1016/j.geomorph.2018.05.021

LEWIS, S.L.; MASLIN, M.A. 2015. Defining the Anthropocene. Nature, 519: 171-180. https:// doi.org/10.1038/nature14258

LI, J.; YANG, L.; PU. R.; LIU, Y. 2017. A review on anthropogenic geomorphology. Journal of Geographical Sciences, 27(1): 109-128. http://dx.doi.org/10.1007/s11442-017-1367-7

MACKLIN, M.G.; LEWIN, J.; JONES, A.F. 2014. Anthropogenic alluvium: an evidencebased meta-analysis for the UK Holocene. Anthropocene, 6: 26-38. http://dx.doi. org/10.1016/j.ancene.2014.03.003

\section{NACSN - NORTH AMERICAN COMISSION ON STRATIGRAPHIC NOMENCLATURE 1983. North American Stratigraphic Code. American Association of Petroleum Geologists Bulletin, 67(5): 841-875.}

OLIVEIRA, A.M.S. 1990. Depósitos tecnogênicos associados à erosão atual. In: ABGE, CONGRESSO BRASILEIRA DE GEOLOGIA DE ENGENHARIA, 6, Salvador, Atas, v.1, p. 411-415.

OLIVEIRA, A.M.S.; PELOGGIA, A.U.G. 2014. The Anthropocene and the Technogene: stratigraphic temporal implications of the geological action of humankind. Quaternary and Environmental Geosciences, 5(2): 103-
111. DOI: http://dx.doi.org/10.5380/abequa. v5i2.34828

OLIVEIRA, A.M.S. 2005. Estudos sobre o Tecnógeno no Brasil. In: ABEQUA, CONGRESSO DA ASSOCIAÇÃO BRASILEIRA DE ESTUDOS DO QUATERNÁRIO, 10, Guarapari (ES), Anais, 6 p.

OLIVEIRA, A.M.S.; BRANNSTRON, C.; NOLASCO, M. C.; PELOGGIA, A.U.G.; PEIXOTO, M.N.O.; COLTRINARI, L. 2005. Tecnógeno: registros da ação geológica do homem. In: C.R.G. Souza, K. Suguio, A.M.S. Oliveira, P.E. Oliveira (orgs.) Quaternário do Brasil. Ribeirão Preto, Holos, p. 363-378.

OLIVEIRA, A.M.S.; PELOGGIA, A.U.G. OLIVEIRA, A.A. 2018. AntropocenoTecnógeno. In: A.M.S. Oliveira\& J.J. Monticelli (eds.) Geologia de Engenharia e Ambiental. São Paulo, ABGE, v. 2, p. 440452.

PELOGGIA, A.U.G. 1995. A dialética da Geologia (temas de Geologia inspirados na obra de Friedrich Engels, e suas aplicações). Revista Brasileira de Geociências, 25(2): 107-110. http://dx.doi.org/10.25249/03757536.1995107110

PElOGgIA, A.U.G. 1997. A ação do homem enquanto ponto fundamental da Geologia do Tecnógeno: proposição teórica básica e discussão acerca do caso do Município de São Paulo. Revista Brasileira de Geociências, 27(3): 257-268. http://dx.doi. org/10.25249/0375-7536.1997257268

PELOGGIA, A.U.G. 1998. O Homem e o ambiente geológico: Geologia, sociedade e ocupação urbana no Município de São Paulo. Xamã, São Paulo, 271 p.

PELOGGIA, A.U.G. 2003. O problema estratigráfico dos depósitos tecnogênicos. In: ABEQUA, CONGRESSO DA ASSOCIAÇÃO BRASILEIRA DE ESTUDOS DO QUATERNÁRIO, 9, Recife (PE), Anais, 5 p.

PELOGGIA, A.U.G. 2004. Sobre a dialética e as particularidades das ciências da natureza e 
da sociedade. Revista Eletrônica Unibero de Produção Científica, março 2004, 16 p.

PELOGGIA, A.U.G. 2015a. Camadas que falam sobre o ser humano, caso encontrem arqueólogos e geólogos que as escutem: rumo a uma arqueogeologia interpretativa dos depósitos tecnogênicos. In: A.M. Ortega, A.U.G. Peloggia (orgs) Entre o Arcaico e $o$ Contemporâneo: ensaios fluindo entre Arqueologia, Psicanálise, Antropologia e Geologia. São Paulo, Iglu, p. 189-221.

PELOGGIA, A.U.G. 2015b. O significado arqueológico dos depósitos tecnogênicoaluviais da Bacia do Rio Una (Taubaté, SP). Revista de Arqueologia Pública, 9(3) [13]: 207-219. https://doi.org/10.20396/rap. v9i3.8641304

PELOGGIA, A.U.G. 2017. O que produzimos sob nossos pés? Uma revisão comparativa dos conceitos fundamentais referentes a solos e terrenos antropogênicos. Revista $U N G$ Geociências, 16(1): 102-127.

PELOGGIA, A.U.G. 2018a. Geological classification and mapping of technogenic (artificial) ground: a comparative analysis. Revista do Instituto Geológico, 39(2): 1 - 15. http://dx.doi.org/10.5935/0100929X.20180005

PELOGGIA, A.U.G. 2018b. The Rock Cycle of the Anthopocene: inserting human agency into the Earth System. Revista do Instituto Geológico, 39(1) 1-13. http://dx.doi. org/10.5935/0100-929X.20180001

PELOGGIA, A.U.G. 2018c. Os registros geológicos da agência humana como categoria temática de patrimônio. In: SBG, SIMPÓSIO NACIONAL DE ENSINO E HISTÓRIA DE CIÊNCIAS DA TERRA, 8, Campinas, Anais, p. 614-620.

PELOGGIA, A.U.G. 2018d. "New Awfull Changes: the human geological stratum rediscovered": allowing art to talk about the Anthropocene. Revista do Instituto Geológico, 39(2): 91-101. http://dx.doi. org/10.5935/0100-929X.20180009

PELOGGIA, A.U.G.; OLIVEIRA, A.M.S. 2005. Tecnógeno: um novo campo de estudos das geociências. In: ABEQUA, CONGRESSO BRASILEIRO DE ESTUDOS DO QUATERNÁRIO, 10, Guarapari, Anais, 4 p.

PELOGGIA, A.U.G.; ORTEGA, A.M. $2016 \mathrm{a}$. Lyell, a agência geológica humana e o Antropoceno: em busca de uma epistemologia geológica e arqueológica. Revista UNG Geociências, 15(2): 106-127.

PELOGGIA, A.U.G.; ORTEGA, A.M. 2016 b. Sobre a recorrência geohistórica de desastres ambientais no Sudeste do Brasil: uma perspectiva de longa duração. Revista $U N G$ - Geociências, 15(2): 61-74.

PELOGGIA, A.U.G.; OLIVEIRA, A.M.S.; OLIVEIRA, A.A.; SILVA, E.C.N.; NUNES, J.O.R. 2014a. Technogenic geodiversity: a proposal on the classification of artificial ground. Quaternary and Environmental Geosciences, 5(1): 28-40. http://dx.doi. org/10.5380/abequa.v5i1.34823

PELOGGIA, A.U.G.; SILVA, E.C.N.; NUNES, J.O.R. 2014b. Technogenic landforms: conceptual framework and application to geomorphologic mapping of artificial ground and landscape as transformed by human geological action. Quaternary and Envorinmental Geosciences, 5(2): 67-81. http://dx.doi.org/ 10.5380/abequa.v5i2.34811

PELOGGIA, A.U.G.; ORTEGA, A.M.; EDGEWORTH, M.; LUZ, R.A. 2017. A expansão do estrato geológico urbano (arqueosfera) no leste do Estado de São Paulo: e relação entre História, Geografia, Geologia e Arqueologia no Antropoceno. Revista Brasileira de Geografia, 62(2): 25-52. https://doi.org/10.21579/issn.25260375_2017_n2_p25-52

ROBB, J. 2005. Agency. In: C. Renfrew\& P. Bahn (eds.) Archaeology: the key concepts. London, Routledge, p. 3-7.

RODRIGUES, C. 2005. Morfologia original e morfologia antropogênica na definição de unidades espaciais de planejamento urbano: exemplo da metrópole paulista. Revista do Departamento de Geografia, 17: 101-111. https://doi.org/10.7154/ RDG.2005.0017.0008 
ROSENBAUM, M.S.; McMILLAN, A.A.; POWELL, J.H.; COOPER, A.H.; CULSHAW, M.G.; NORTHMORE, K.J. 2003. Classification of artificial (manmade) ground. Engineering Geology, 69: 399-409. http://dx.doi.org/10.1016/S00137952(02)00282-X

ROSS, J.L.S. 1992. O registro cartográfico dos fatos geomórficos e a questão da taxonomia do relevo. Revista do Departamento de Geografia, 6: 17-29.

RUDDIMAN, W.F. 2013. The Anthropocene. Annual Review of Earth and Planetary Sciences 41: 45-68. https://doi.org/10.1146/ annurev-earth-050212-123944

RULL,V.2016.TheHumanizedEarthSystem(HES). The Holocene, 26(9): 1513-1516. https://doi. org/10.1177\%2F0959683616640053

SCHIFFER, M.B. 1996. Formation processes of the archaeological record. University of Utah Press, Salt Lake City, 428 p.

STEFFEN, W.; GRINEVALD, J.; CRUTZEN, P.; McNEILL, J. 2011. The Anthropocene: conceptual and historical perspectives. Philosophical Transactions of the Royal Society A, 369: 842-867. https://doi. org/10.1098/rsta.2010.0327

STEFFEN, W.; LEINFELDER, R.; ZALASIEWICZ, J.; WATERS, C.N.; WILLIAMS, M.; SUMMERHAYES, C.; BARNOSKY, A.D.; CEARRETA, A.; CRUTZEN, P.; EDGEWORTH, M.; ELLIS, E.; FAIRCHILD, I,J.; GALUSZKA, A.; GRINEVALD, J.; HAYWOOD, A.; IVAR DO SUL, J.; JEANDEL, C.; McNEILL, J.R.; ODADA, E.; ORESKES, N.; REVKIN, A.; RICHTER, D. de B.; SYVITSKI, J.; VIDAS, D.; WAGREICH, M.; WING, S.L.; WOLFE, A.P.; SCHELLNHUBER, H.J. 2016. Stratigraphic and Earth System approaches to defining the Anthropocene. Earth's Future, 4: 324-345. https://doi. org/10.1002/2016EF000379

STINCHCOMB， G.E.; STEWART, R.M.; MESSNER, T.C.; NORDT, L.C.; DRIESE, S.G.; ALLEN, P.A. 2013. Using event stratigraphy to map the Anthropocene - an example from the historic coal mining region in eastern Pennsylvania, USA. Anthropocene, 2: 42-50. http://dx.doi.org/10.1016/j. ancene.2013.06.001

TAROLLI, P.; SOFIA, G. 2016. Human topographic signatures and derived geomorphic processes across landscapes. Geomorphology, 255: 140-161. http://dx.doi.org/10.1016/j. geomorph.2015.12.007

TER STEPANIAN, G. 1988. Beginning of the Technogene. Bulletin of the International Association of Engineering Geology, 38: 133-142.

UNDERWOOD, J.R. 2001a. Anthropic rocks: made, modified and moved by humans. GSA Today, 11(11): 19.

UNDERWOOD, J.R. 2001b. Anthropic rocks as a fourth class. Environmental \& Engineering Geoscience, 7(1): 104-110. DOI: https://doi. org/10.2113/gseegeosci.7.1.104

WATERS, C.N. 2018. Artificial ground. In: P.T. Bobrowsky \& B. Marker (eds.) Encyclopaedia of Engineering Geology. Cham, Springer International, p. 1-15.

WATERS, C.N.; ZALASIEWICZ J. 2018. Concrete: the most abundant novel rock type of the Anthropocene. In: D.A. Della Sala \& M.I. Goldstein (eds) The Encyclopaedia of the Anthropocene, Elsevier, Oxford, v.1, p. 75-85. https://doi.org/10.1016/B978-0-12809665-9.09775-5

WATERS, C.N.; ZALASIEWICZ, J.A.; WILLIAMS, M.; ELLIS, M.A.; SNELLING, A.M. 2014. A stratigraphical basis for the Anthropocene? In: C.N. Waters; J.A. Zalasiewicz; M. Williams; M. Ellis; A.M. Snelling (eds.). A stratigraphical basis for the Anthropocene. London Geological Society, Special Publication 395, p. 1-21. https://doi. org/10.1144/SP395.18

WATERS, C.N.; FAIRCHILD, I.J.; McCARTHY, F.M.G.; TURNEY, C.S.M.; ZALASIEWICZ, J.; WILLIAMS, M. 2018. How to date natural archives of the Anthropocene. Geology Today, 34(5): 182-187. https://doi. org/10.1111/gto.12245

WILLIAMS, M.; ZALASIEWICS, J.; WATERS, C.; HIMSON, S.; SUMMERHAYES, 
C.; BARNOSKY, A.; LEINFELDER, R. 2018. The paleontological record of the Anthropocene. Geology Today, 34(5): 188193. https://doi.org/10.1111/gto.12246

ZALASIEWICZ, J. 2008. The Earth after us: what legacy will humans leave in the rocks? Oxford University Press, Oxford, $251 \mathrm{p}$.

ZALASIEWICZ, J.; WILLIAMS, M.; FORTEY, R.; SMITH, A.; BARRY, T.L.; COE, A.L.; BOWN, P.R.; RAWSON, P.F.; GALE, A.; GIBBARD, P.; GREGORY, F.J.; HOUNSLOW, M.W.; KERR, A.C.; PEARSON, P.; KNOX, R.; POWELL, J.; WATERS, C.; MARSHALL, J.; OATES, M.; STONE, P. 2011. Stratigraphy of the Anthropocene. Philosophical Transactions of the Royal Society A, 369: 1036-1055. https:// doi.org/10.1098/rsta.2010.0315

ZALASIEWICZ. J.; WILLIAMS, M.; WATERS, C.N.; BARNOSKY, A.D.; HAFF, P. 2014a. The technofossil record of humans. The Anthropocene Review, 1(1): 34-43. https:// doi.org/10.1177\%2F2053019613514953

ZALASIEWICZ, J.; KRYZA, R.; WILLIAMS, M. 2014b. The mineral signature of the Anthropocene in its deep-time context. In: C.N. Waters; J.A. Zalasiewicz; M. Williams; M.A. Ellis; A.M. Snelling (eds.) $A$ stratigraphical basis for the Anthropocene. London, Geological Society, (Special Publication 395), p. 109-117. https://doi. org/10.1144/SP395.2

ZALASIEWICZ, J.; WATERS, C.N.; IVAR DO SUL, J.A.; CORCORAN, P.L.; BARNOSKY, A.D.; CEARRETA, A.; EDGEWORTH, M.; GAHISKA, A.; JEANDEL, C.; LEINFELDER, R.; MCNEILL, J.R.; STEFFEN, W.; SUMMERHAYES, C.; WAGREICH, M.; WILLIAMS, M.; WOLFE, A.P.; YONAN, Y. 2016. The geological cycle of plastics and their use as a stratigraphic indicator of the Anthropocene. Anthropocene, 13: 4-17. https://doi. org/10.1016/j.ancene.2016.01.002

ZALASIEWICZ, J.; WATERS, C.; WILLIAMS, M. 2017a. Les strates de la ville de l'Anthropocène. Annales: Histoire, Sciences Sociales, 72(2): 329-351. https://doi. org/10.1017/S0395264917000567
ZALASIEWICZ, J.; WATERS, C.N.; SUMMERHAYES, C.P.; WOLFE, A.P.; BARNOSKY, A.D.; CEARRETA, A.; CRUTZEN, P.; ELLIS, E.; FAIRCHILD, I.J.; GALUSZKA, A.; HAFF, P.; HADJAS, I.; HEAD, M.J.; IVAR DO SUL, J., JEANDEL, C.; LEINFELDER, R.; McNEILL, J.R.; NEAL, C.; ODADA, E.; ORESKES, N.; STEFFEN, W.; SYVITKSKI, J.; VIDAS, D.; WAGREICH, M.; WILLIAMS, M. 2017 b. The Working Group on the Anthropocene: summary of evidence and interim recommendations. Anthropocene, 19: 55-60. https://doi.org/10.1016/j.ancene.2017.09.001

ZALASIEWICZ, J.; WATERS, C.N.; WOLFE, A.P.; BARNOSKY, A.D.; CEARRETA, A.; EDGEWORTH, M.; ELLIS, E.C.; FAIRCHILD, I.J.; GRADSTEIN, F.M.; GRINEVALD, J.; HAFF, P.; HEAD, M.J.; IVAR DO SUL, J.A.; JEANDEL, C.; LEINFELDER, R.; McNEILL, J.R.; ORESKES, N.; POIRIER, C.; REVKIN, A.; RICHTER, D. deB.; STEFFEN, W.; SUMMERHAYES, C.; SYVITSKI, J.P.M.; VIDAS, D.; WAGREICH, M.; WING, S.; WILLIAMS, M. 2017c. Making the case for a formal Anthropocene Epoch: an analysis of ongoing critiques. Newsletters on Stratigraphy, 50(2): 205-226. https://doi. org/10.1127/nos/2017/0385

ZALASIEWICZ J.; WILLIAMS, M.; WATERS, C.N.; BARNOSKY A.D.; PALMESINO, J.; RÖNNSKOG, A.-F.; EDGEWORTH, M.; NEAL, C.; CEARRETA, A.; ELLIS, E.C.; GRINEVALD, J.; HAFF, P.; IVAR DO SUL, J.A.; JEANDEL, C.; LEINFELDER, R.; McNEILL, J.R.; ODADA, R.; ORESKES, N.; PROCE, S.J.; REVKIN, A.; STEFFEN, W.; SUMMERHAYES, C.; VIDAS, D.; WING, S.; WOLFE, A.P. 2017d. Scale and diversity of the physical technosphere: a geological perspective. The Anthropocene Review, 4(1): 9-22. https://doi. org/10.1177\%2F2053019616677743

ZALASIEWICZ, J.; WATERS, C.N.; SUMMERHAYES, C; WILLIAMS, M. 2018a. The Anthropocene. Geology Today, 34(5): 177-181. https://doi.org/10.1111/ gto. 12244 
ZALASIEWICZ, J.; WATERS, C.; WILIAMS, M.; ALDRIDGE, D.C.; WILKINSON, I,P. 2018b. The Stratigraphical signature of the Anthropocene in England and its wider context. Proceedings of the Geologist's Association, 129(3): 482-491. https://doi. org/10.1016/j.pgeola.2017.06.004

ZALASIEWICS, J.; WATERS, C.N.; WILLIAMS, M.; SUMMERHAYES, C. (eds.) 2019a. The Anthropocene as a geological time unit: a guide to the scientific evidence and current debate. Cambridge, Cambridge University Press, 362 p.
ZALASIEWICZ, J.; WATERS, C.N.; HEAD, M.J.; POIRIER, C.; SUMMERHAYES, C.P.; LEINFELDER, R.; GRINEVALD, J.; STEFFENS, W.; SYVITSKI, J.P.M.; HAFF, P.; McNEILL, J.R.; WAGREICH, M.; FAIRCHILD, I.J.; RICHTER, D.D.; VIDAS, D.; WILLIAMS, M.; BARNOSKY, A.D. 2019b. A formal Anthropocene is compatible with but distinct from its diachronous anthropogenic counterparts: a response to W.F. Ruddiman's 'three-flaws in defining a formal Anthropocene'. Progress in Physical Geography (no prelo). https://doi. org/10.1177\%2F0309133319832607

\section{Endereço do autor:}

Alex Ubiratan Goossens Peloggia - Av. São Luís 71, ap. 304, CEP: 01046-001, São Paulo, SP, Brasil. E-mail: alexpeloggia@uol.com.br

Artigo submetido em 13 de fevereiro de 2019, aceito em 22 de março de 2019. 